\title{
Introdução à Geometria Fractal no Ensino Médio Técnico: Uma Abordagem com Programação Python
}

\author{
Gustavo Vieira Ferreira \\ Instituto Federal Catarinense - IFC \\ Araquari, SC, Brasil \\ gusferreira1203@gmail.com
}

\author{
Weliton Dal Pizzol Maria \\ Instituto Federal Catarinense - IFC \\ Araquari, SC, Brasil \\ welitondpm2003@gmail.com
}

\author{
Adriano Rodrigues de Melo \\ Instituto Federal Catarinense - IFC \\ Araquari, SC, Brasil \\ adriano.melo@ifc.edu.br
}

\begin{abstract}
This work is inserted in the context of technical high school and it aimed to analyze the integration between the branches of Fractal Geometry, Analytical Geometry and Computer Programming. For this purpose, we carried out a bibliographic search about what characterizes and distinguishes Fractal Geometry from Euclidean Geometry, we also seek in our readings to list the most famous fractals. Then, we developed (in python language) several fractal generation programs. It was possible to work with amazing and easily programmable fractal shapes, such as the Cantor Set, the Hilbert Curve and Sierpinski Triangle. We also built two new families of fractal shapes from a generalization of the Koch Curve. We conclude that programming fractals in the context of technical high school is productive and challenging, as it requires many changes in the representations of fractal patterns.
\end{abstract}

\section{KEYWORDS}

Geometria no Ensino Médio Técnico, Interdisciplinaridade, Programação.

\section{INTRODUÇÃO}

O ensino de Geometria no Ensino Médio trata das formas planas e tridimensionais e suas representações em desenhos, planificações, modelos e objetos do mundo concreto. No entanto, este estudo se limita tradicionalmente à Geometria Euclidiana que, ao considerar objetos planos e tridimensionais regulares, não é suficiente para representar o mundo físico. De fato, a grande maioria das formas geométricas físicas consistem em objetos não planos e irregulares. Qual a forma de uma couve-flor, de uma árvore ou da costa marítima brasileira? Certamente não é uma semiesfera, um cilindro ou um conjunto de segmentos de reta, respectivamente. A observação de que a natureza é irregular fomentou a origem das geometrias conhecidas como Não Euclidianas, tais como a esférica, a elíptica, a hiperbólica e a fractal.

A partir deste cenário, vários trabalhos foram realizados no sentido de se inserir tópicos de Geometria Não Euclidiana no âmbito escolar, tais como os trabalhos de [1-7] e vários outros que apresentam metodologias, estratégias e atividades de inserção combinando elementos que relacionam geometria plana e espacial com os conceitos das Geometrias Não Euclidianas consideradas.

Este trabalho apresentará uma outra abordagem para o estudo inicial de fractais, que inclui, além da análise espacial, elementos de Geometria Analítica e Programação de Computadores. Embasados pela característica recursiva dos fractais, isto é, pelo fato de sua estrutura "global" ou "final" ser definida pela repetição infinita de um padrão geométrico "local" ou "inicial”, formula-se a hipótese de que os (ou pelo menos alguns dos) fractais produzidos pela mente humana (o Conjunto de Cantor, por exemplo) podem ser trabalhados, no nível do ensino médio, com Computação e elementos de Geometria Analítica Plana.

Desenvolveu-se, nesta linha, um projeto extra-classe com dois estudantes do ensino médio técnico de informática, com o objetivo de investigar as formas fractais à luz da Computação e da Geometria Analítica Plana. Também se teve por intenção coletar informações acerca das potencialidades e fragilidades dessa abordagem.

\section{REVISÃO DE LITERATURA}

O termo fractal foi primeiramente empregado pelo matemático francês Benoit B. Mandelbrot (1977): "Eu cunhei o termo fractal do adjetivo latino fractus. $\mathrm{O}$ verbo latino frangere correspondente, significa 'quebrar': para criar fragmentos irregulares" [8, p. 4].

"Frangere para criar fragmentos irregulares" a fim de estabelecer uma ferramenta capaz de representar mais adequadamente e realisticamente os objetos da natureza, uma vez que a Geometria Euclidiana se torna muito limitada para tal propósito: montanhas, árvores, nuvens ou folhas são exemplos de formas da natureza que não podem ser expressos, de uma maneira satisfatoriamente realística, em termos da geometria clássica.

Os fractais são caracterizados principalmente pela auto semelhança, por sua complexidade infinita e por sua dimensão [9]. Considere, para fins de discussão de suas propriedades gerais, o exemplo do fractal conhecido como Conjunto de Cantor, cuja construção é apresentada na Figura 1 e se inicia (nível $n=0$ ) com um segmento de reta $l_{0}$ de comprimento $\varepsilon=L$. O próximo passo de sua construção consiste em dividir o segmento $l_{0}$ em três partes de mesmo comprimento e excluir sua parte central, gerando assim os segmentos do nível $n=1: l_{1}$, formado de duas partes $(N=2)$, cada qual com comprimento $\varepsilon=L / 3$. Repete-se esse processo nos demais níveis: divide-se cada segmento do nível anterior em três partes de mesmo tamanho e excluí-se aquele que ocupa a posição central, como demonstrado pela Figura 1.

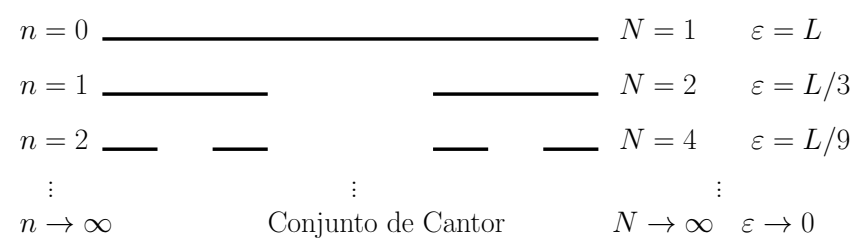

Figure 1: Níveis de construção do fractal Conjunto de Cantor, sendo que o número de segmentos e seus respectivos comprimentos no $n$-ésimo nível é dado, respectivamente, por $N=2^{n}$ e $\varepsilon=L / 3^{n}$. 
Observe que o processo recursivo no qual o Conjunto de Cantor foi construído pode ser repetido indefinidamente. Isso implica que é virtualmente impossível obter uma imagem final desse fractal, uma vez que ele é o limite $(n \rightarrow \infty)$ desse processo recursivo. Essa característica é o que se denomina por complexidade infinita $[10, \mathrm{p}$ 31].

A propriedade da auto semelhança ocorre quando um objeto apresenta o mesmo aspecto visual em todas as escalas [9] e ela pode ser exata ou aproximada (também chamada de estatística). Em geral, fractais gerados por processos matemáticos são caracterizados por uma auto semelhança exata (é o caso do conjunto de Cantor, conforme Figura 2), enquanto que a auto-semelhança estatística é observada nas formas da natureza (veja Figura 3).
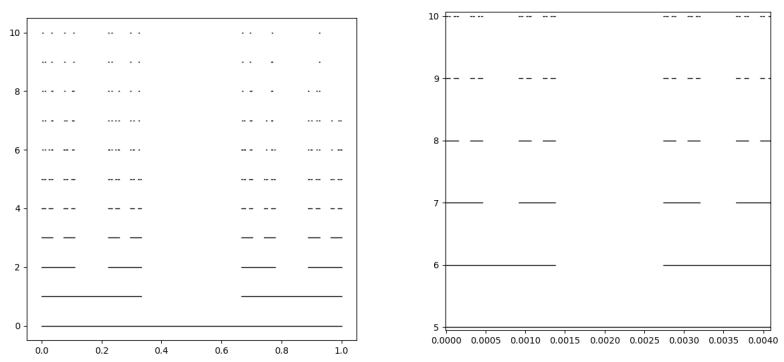

Figure 2: A esquerda, os 10 primeiros níveis do Conjunto de Cantor. A direita, uma ampliação desses níveis evidenciando a propriedade da auto-semelhança: ao reduzir-se a escala, encontra-se uma "cópia dele mesmo".
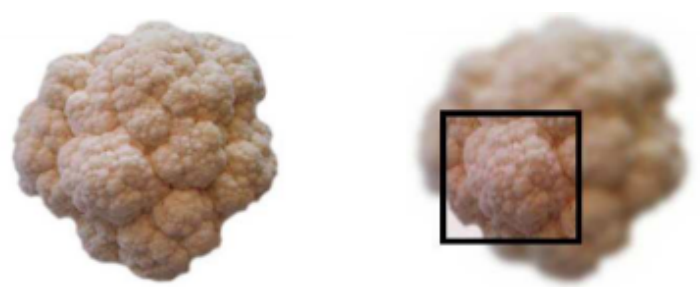

Figure 3: A esquerda, uma couve-flor e a direita uma réplica observada: uma cópia aproximada da couve-flor em escala reduzida (Fonte: [10, p. 33]).

Na Figura 1 foi registrado que o número total $N$ de segmentos no $n$-ésimo nível é $2^{n}$, que passa a ser indefinidamente grande quando $n$ é também muito grande, em outras palavras, $N$ se torna ilimitado quando $n$ tende ao infinito. Por outro lado, o comprimento total dos segmentos, dado por $N \varepsilon=2^{n}\left(L / 3^{n}\right)$, ou equivalentemente, $L(2 / 3)^{n}$, se aproxima de zero quando $n$ tende ao infinito.

Esses dois resultados são um tanto contraditórios: eles dizem que o Conjunto de Cantor é constituído por infinitos segmentos e que, no entanto, totalizam um comprimento nulo. Essa contradição aparente surge porque a experiência com Geometria Euclidiana sugere que segmentos de reta é o que tem comprimento (dimensão 1), enquanto que ponto é o que não tem grandeza alguma (dimensão 0). Poderia-se cogitar que essa "poeira de Cantor" esteja "situada" numa dimensão intermediária às dimensões 0 e 1 ? Parece absurda essa hipótese, porém, esse é exatamente o caso.

Com efeito, seja um conjunto $A$ de pontos num espaço de dimensão $p$. Define-se a dimensão fractal (ou dimensão de HausdorffBesicovitch) como [11, p. 254]:

$$
D=\lim _{\varepsilon \rightarrow 0} \frac{\log N(\varepsilon)}{\log (1 / \varepsilon)}
$$

em que $N(\varepsilon)$ é o número de hipercubos de lado $\varepsilon$ necessários para cobrir todo o conjunto de pontos $A$. No caso do Conjunto de Cantor, os hipercubos podem ser os próprios segmentos de reta e concluise, dessa forma, que $N(\varepsilon)=2^{n}$ e $\varepsilon=L / 3^{n}$. Observando que fazer $\varepsilon \rightarrow 0$ é o mesmo que fazer $n \rightarrow \infty$, pode-se calcular a dimensão desse fractal aplicando-se a fórmula (1):

$$
D=\lim _{n \rightarrow \infty} \frac{\log 2^{n}}{\log \left(3^{n} / L\right)}=\lim _{n \rightarrow \infty} \frac{\log 2}{\log 3-(\log L) / n}=\frac{\log 2}{\log 3} \approx 0,63 .
$$

Conclui-se com isso, intuitivamente, que o conceito de dimensão fractal está estreitamente relacionado com a estrutura de ocupação de espaço da figura [9].

\section{MATERIAIS E MÉTODOS}

Esta pesquisa é classificada, quanto aos objetivos, em exploratória pois, conforme [12], pesquisas exploratórias têm como principal objetivo o aprimoramento de ideias ou a descoberta de intuições. De fato, este trabalho esteve alicerçado na investigação de estruturas fractais em nível computacional e foi apoiado por conceitos elementares da Geometria Analítica (GA) constantes em livros do ensino médio. Nestes termos, fez parte do processo de investigação o descobrimento de quais conceitos (de GA e de Programação) seriam necessários, quais fractais seriam mais adequados ou possíveis de serem abordados pela proposta, além de quais compreensões e dificuldades estariam em jogo nas conversões de registros.

Quanto aos procedimentos, caracteriza-se como uma pesquisa bibliográfica, uma vez que a pesquisa bibliográfica é aquela que se utiliza de dados ou categorias teóricas já trabalhadas por outros pesquisadores e devidamente registradas [13, p. 118]. Segundo [14], a pesquisa bibliográfica não é mera repetição do que já foi dito ou escrito sobre certo assunto, mas propicia o exame de um tema sob novo enfoque ou abordagem, chegando a conclusões inovadoras. A novidade do presente trabalho está justamente no enfoque: o computacional.

O projeto teve inicio com uma pesquisa bibliográfica acerca: i) das principais diferenças entre as Geometrias Euclidiana e Fractal e, por tanto, o que define esta última; ii) dos principais fractais da literatura, também chamados de fractais clássicos, por serem os predecessores dessa área; iii) dos principais conceitos associados à Geometria Analítica Plana, a saber: sistema cartesiano ortogonal, conceitos de ponto, reta, segmento de reta, trigonometria e polígonos. O ponto i) foi descrito na seção 2, enquanto que os fractais utilizados neste trabalho, referidos pelo item ii), foram descritos na seção 4 . Os conceitos que se referem ao terceiro ponto foram sendo requisitados no decorrer da pesquisa, conforme se avançava na programação. Após a seleção dos fractais, os esforços foram direcionados para a elaboração de mecanismos computacionais capazes de exprimir seus padrões, ou seja, além do padrão geométrico/visual que determina a figura fractal, procurava-se também o padrão algébrico que o 
constitui. Esse padrão algébrico foi, por último, programado na linguagem de programação python. A última etapa foi destinada ao estudo da dimensão fractal.

\section{RESULTADOS E DISCUSSÕES}

Foram estudados diversos fractais e construiu-se, para cada qual, um programa gerador. A Figura 4 dispõe as imagens produzidas por alguns desses programas ${ }^{1}$.
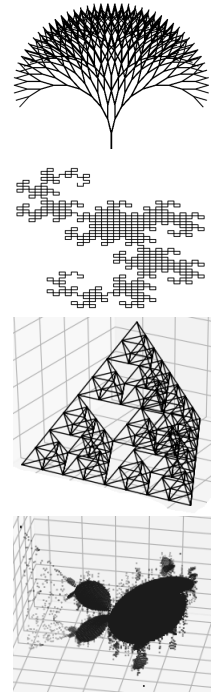

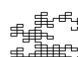

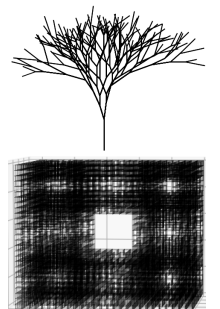
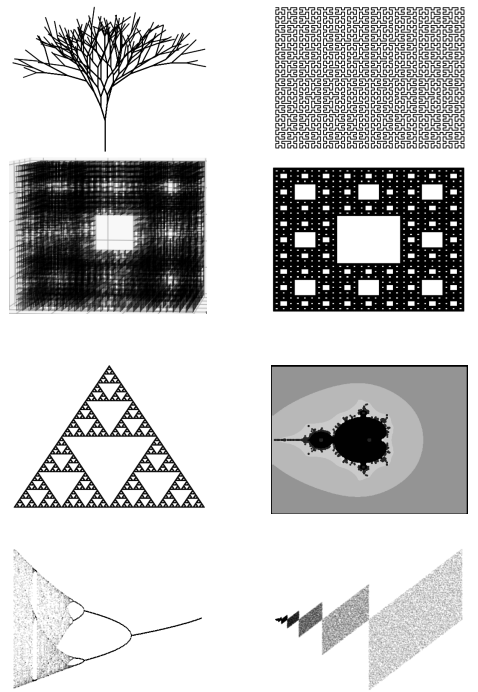
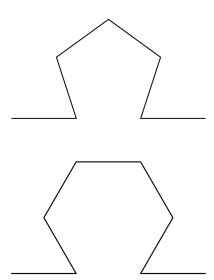

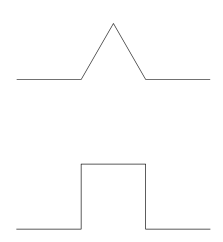

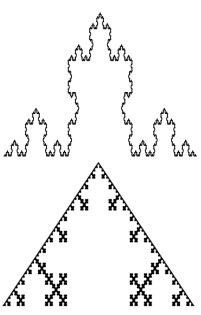

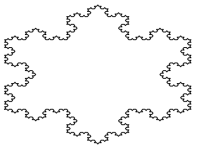<smiles>C1C2C3CC4C5C3C1C1C2C4C51</smiles><smiles>C1CC(C2CCCC2C2CCC2)C1</smiles>

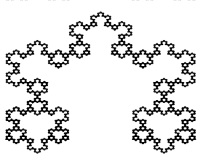

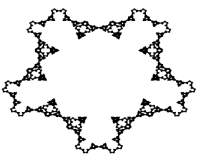

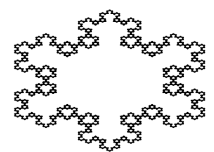

Figure 5: Sementes ( $1^{\text {a }}$ coluna) e respectivas curvas de Koch ( $2^{\mathrm{a}}$ coluna) e flocos de Koch ( $3^{\mathrm{a}}$ coluna), construídas para $n=$ $3,4,5$ e 6 (a curva padrão é obtida ao considerar-se $n=3$ ).

Figure 4: Alguns dos fractais produzidos ao longo da execução do projeto; $1^{\text {a }}$ Coluna: Árvore Binária (sem ruído), Curva do Dragão, Tetraedro de Sierpinski, Conjunto de Man-

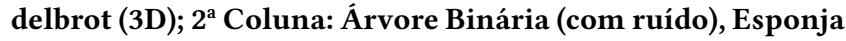
de Menger, Triângulo de Sierpinski, Mapa Logístico; $3^{\text {a }} \mathrm{Col}$ una: Curva de Hilbert, Tapete de Sierpinski, Conjunto de Mandelbrot, Primos Binários.

O fractal conhecido como Curva de Koch foi explorado com algum detalhe. Originalmente ele tem uma semente de quatro segmentos definida conforme Figura $5\left(1^{\circ}\right.$ gráfico da $1^{\text {a }}$ coluna: semente $\operatorname{com} n=3$ ). Elaborou-se uma família de fractais a partir de uma modificação de sua semente: tomando $k$ segmentos congruentes no lugar de 4 (veja Figura 5). Constatou-se que curvas de Koch com $n \geq 5$ possuem sobreposição de segmentos de reta. O Floco de Koch, por ser construído de maneira análoga à curva de Koch, também pôde ser generalizado, conforme mostrado na Figura $5,3^{\text {a }}$ coluna.

O Triângulo de Sierpinski também foi extensamente explorado. A forma mais intuitiva de construí-lo é aquela em que se remove os triângulos (semelhantes) de suas áreas remanescentes. Esse método, chamado de poligonal (ver Figura $6,1^{\text {a }}$ coluna), se mostrou bastante custoso computacionalmente, principalmente porque o algoritmo desenvolvido realiza uma contagem de caixas para áreas por meio de loops aninhados para percorrer listas (algo conhecido por exigir bastante do processador). Outro método empregado foi

${ }^{1}$ Os códigos dos programas geradores desses e de outros fractais estão disponíveis no repositório: https://github.com/Welitondpm/Projeto-Fractal.
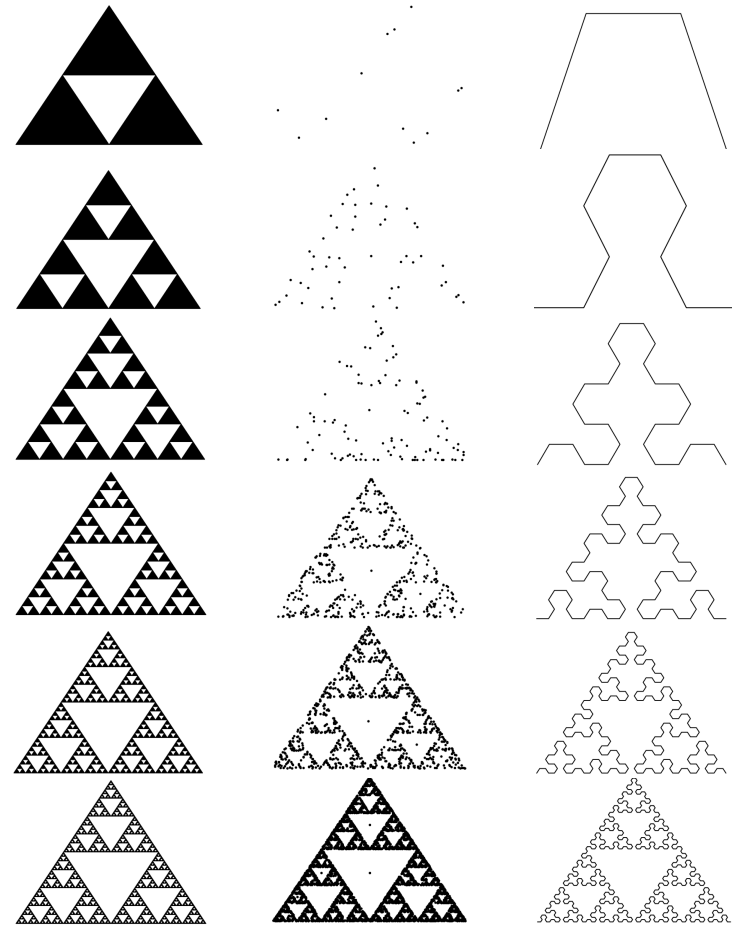

Figure 6: Algumas iterações das três formas de se obter o Triângulo de Sierpinski: método poligonal (esquerda), método caótico (centro) e método linear (direita).

aquele baseado na aleatoriedade: o padrão do Triângulo de Sierpinski surge ao, partindo de três pontos (vértices de um triângulo equilátero), registrar-se os pontos médios entre um ponto inicial qualquer (escolheu-se o baricentro) e um dos vértices do triângulo, vértice este obtido por sorteio. Essa escolha, mesmo sendo aleatória, preenche de forma efetiva os pontos do dito fractal, mesmo que o 
ponto inicial não esteja contido no mesmo (que é o caso do baricentro), o padrão do Triângulo de Sierpinski se torna mais evidente conforme as iterações aumentam. Imagens desse processo são mostradas na Figura 6, $2^{\text {a }}$ coluna.

A terceira forma gera um Triângulo de Sierpinski com um padrão determinístico e com uma performance muito superior. Tal método foi denominado de linear e também é conhecido como Sierpinski Arrowhead, ou, em tradução livre, Ponta de Flecha de Sierpinski. Ele tem uma semente linear (veja Figura $6,3^{\mathrm{a}}$ coluna de gráficos) que é aplicada em segmentos, sendo portanto uma curva. Constatou-se que, em geral, curvas são bem mais baratas computacionalmente que polígonos, porque as curvas podem muito facilmente ser operadas com todos os pontos listados na mesma variável, precisando, portanto, de menos loops para concluir a iteração.

Descreveu-se aqui apenas dois dos diversos fractais estudados. Ressalte-se, além disso, que também foi desenvolvida uma função que computa aproximações da dimensão fractal pelo método da contagem de caixas (veja [11] para detalhes sobre esse método).

\section{CONSIDERAÇÕES FINAIS}

Este trabalho buscou apresentar alguns resultados obtidos a partir de um projeto extra-classe desenvolvido com o objetivo de investigar a viabilidade de se introduzir elementos de Geometria Fractal, junto ao ensino médio técnico, de modo associado à Geometria Analítica e à Programação de Computadores. Fez-se, para este fim, uma pesquisa bibliográfica acerca daquilo que caracteriza e distingue a Geometria Fractal da Geometria Euclidiana. Uma lista contendo os mais famosos fractais (aqueles que apareceram com maior frequência na pesquisa) foi produzida. A partir dessa lista, empreendeu-se a jornada de construir funções computacionais capazes de os gerar em um nível arbitrário.

Conforme já mencionado, foi grande a diversidade de fractais trabalhados, de modo que o espectro de conceitos utilizados também foi amplo, o que por outro lado, fugiu um pouco do planejado, uma vez que se esperava utilizar apenas conceitos de geometria analítica plana. De fato, adentrou-se não apenas no domínio tridimensional, como também no campo dos números complexos, da teoria dos números e da probabilidade (veja Figuras 4 e 6).

Ressalte-se que foi possível trabalhar com formas incríveis mesmo a partir de ferramentas simples. Cite-se os casos do conjunto de Cantor, da curva de Hilbert e do triângulo de Sierpinski (gerado por processos aleatórios). Os principais conceitos envolvidos na construção deste último fractal foram: representação de pontos, ponto médio e noções de aleatoriedade (sua programação se mostrou muito simples). Também foi possível extrapolar o padrão da Curva de Koch, ao construir-se duas famílias de novas formas fractais a partir de uma generalização de sua semente (Figura 5).

Importante registar também que o trabalho envolvido na programação dos fractais exigiu um certo esforço, pois se viu dependente de diversas transformações de representação e identificação de padrões (tanto geométricos quanto computacionais): a figura fractal, ponto de partida do processo, já possui a complexidade de ser construída por iteração (os níveis); esse padrão geométrico precisa ser representado por meio de relações (a maioria são segmentos de reta, de modo que os vértices desses segmentos é quem precisam ser representados); essas relações precisam ser generalizadas, isto é, precisa-se identificar o seu padrão; por fim, há que se realizar uma nova conversão de registro: converter o padrão algébrico encontrado para uma linguagem computacional.

Conclui-se, nestes termos, que a introdução de elementos de Geometria Fractal, junto ao ensino médio técnico, de modo associado à Geometria Analítica e à Programação de Computadores pode ser viável, de modo que se percebeu um grande potencial para essa abordagem interdisciplinar, com problemas dos mais simples até os mais avançados.

Tal afirmação encontra respaldo no fato de que se obteve êxito com a execução desse projeto extra-classe, o que é comprovado pela variedade de programas construídos (todos desenvolvidos pelos próprios estudantes!) e de fractais abordados. Além disso, a conexão entre o ramo da Geometria Analítica e a Computação se viu inevitável à medida em que o projeto avançava. Mesmo as mais simples formas fractais envolveram representações de pontos e segmentos de reta no sistema cartesiano ortogonal, além de cálculos de distâncias, medições de ângulos, transformações de rotações e translações.

Ressalte-se, por fim, que houveram duas situações que ofereceram um grande apoio para a execução do projeto: primeiro, as atividades foram desenvolvidas na forma de projeto extra-classe, o que implica que havia mais tempo para reflexão e descobrimentos; e, segundo, foram desenvolvidas com estudantes do Curso Técnico em Informática Integrado ao Ensino Médio, o que implica que conhecimentos sobre programação não foram uma preocupação.

\section{AGRADECIMENTOS}

O presente trabalho foi realizado com o apoio do Instituto Federal Catarinense, Campus Araquari, nos termos do Edital n 16/2019.

\section{REFERÊNCIAS}

[1] A. B. Bergmann and C. A. H. Nicolini. A geometria fractal na sala de aula uma experiência. In X Encontro Nacional de Educação Matemática, Salvador, Jul. 2010.

[2] D. G. Cruz and C. H. Santos. Algumas diferenças entre a geometria euclideana e as geometrias não-euclideanas hiperbólica e elíptica - a serem abordadas nas séries do ensino médio. In X Encontro Paranaense de Educação Matemática, Paraná, Set. 2009.

[3] R. W. S. Faria and M. V. Maltempi. Padrões fractais: conectando matemática e arte. EccoS Rev. Cient., (27):33 - 53, 2012.

[4] R. A. Fuzzo, T. S. Santos, and L. Ferreira. Fractais e o geogebra: construindo a curva de koch. In XIII Conferência Interamericana de Educação Matemática, Recife, Jun. 2011.

[5] M. Nascimento, S. C. R. Silva, and N. A. Maciel. Uma proposta didática para o ensino de geometria fractal em sala de aula na educação básica. VIDYA, 32(2):113 $-132,2012$.

[6] A. S. Pereira. Fractais circulares: algumas considerações e atividades. Master's thesis, Universidade Estadual de Londrina, Londrina, 2013.

[7] R. M. Barbosa. Descobrindo a geometria fractal para a sala de aula. Ed Autêntica, Belo Horizonte, 2005

[8] B. B. Mandelbrot. The fractal geometry of nature. W. H. Freeman and Company, New York, 1977.

[9] Thiago Albuquerque de Assis, José Garcia Vivas Miranda, Fernando de Brito Mota, Roberto Fernandes Silva Andrade, and Caio Mário Castro de Castilho. Geometria fractal: propriedades e características de fractais ideais. Revista Brasileira de Ensino de Física, 30:2304.1 - 2304.10, 2008. ISSN 1806-1117.

[10] R. S. R. Nunes. Geometria fractal e aplicações. Master's thesis, Departamento de Matemática Pura. Faculdade de Ciências da Universidade do Porto, Porto, Portugal, 2006.

[11] N. Fiedler-Ferreira and C. P. C. Prado. Caos - uma introdução. Blucher, São Paulo, 1994.

[12] A. C. Gil. Como Elaborar Projetos de Pesquisa. Atlas, São Paulo, 2002.

[13] A. J. Severino. Metodologia do trabalho científico. Cortez, São Paulo, 2007.

[14] E. M. Lakatos and M. A. Marnoni. Fundamentos de Metodologia Científica. Atlas, São Paulo, 2003. 